Prof. Dr. Heiner Rindermann

Pädagogische und Entwicklungspsychologie TU Chemnitz

heiner.rindermann@psychologie.tu-chemnitz.de www.tu-chemnitz.de/ hrin
20. Fachgruppentagung Entwicklungspsychologie, Universität Erfurt, 12.-14. September 2011, Nordhäuser Str. (63)

(Prof. Dr. Bärbel Kracke \& B.A. Vivien Scholze) AG 30, Dienstag, 13.9.11, Lehrgebäude 1, Raum 218

Leiter HR, 11.00-12.45 Uhr HR 11.00-11.15-11.20 Uhr

\title{
Heiner Rindermann
}

TU Chemnitz

\section{Intelligenzwachstum in Kindheit und Jugend}




\section{Gliederung}

1 Theoretische Annahmen ..............................................3

2 Probleme der Forschung ........................................7

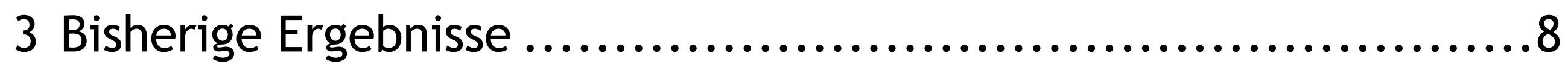

4 Testauswertungen............................................. 10

5 Querschnittliche Studien...................................... 13

6 Längsschnittliche Studie ....................................... 18

7 Diskussion und Ausblick ............................................. 21 


\section{Theoretische Annahmen}

Piagets Stadientheorie

Verbesserung des Denkens (richtiger, intelligenter, rationaler werdend) durch selbständige Auseinandersetzung mit Umwelt. 


\section{Psychometrie - Cattell}

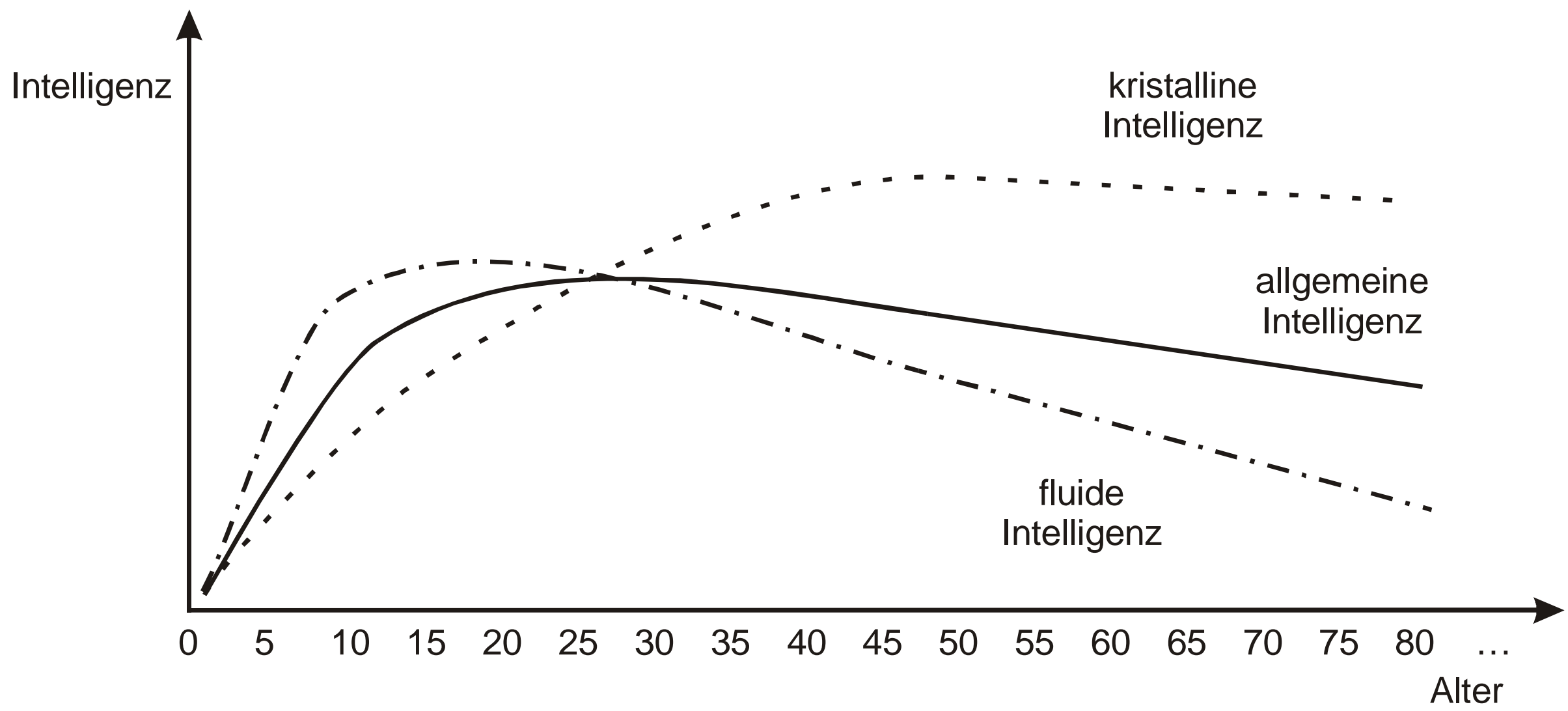

Fluide Intelligenz wächst durch biologische Reifungsprozesse. Kristalline Intelligenz wächst durch investierte fluide und Umwelt (Lernen). 


\section{Psychometrie - Informationsverarbeitungsgeschwindigkeit}

Mental Speed erhöht Arbeitsgedächtniskapazität und beide erhöhen zusammen Intelligenz (z.B. Jensen). Biologische Reifung.

Wissen - Expertise- und Schulleistungsforschung

Umfangreichere und besser strukturierte Wissensbasis (z.B. Ericsson). 
Alle nehmen an, daß Intelligenz ansteigt, aber um wieviel pro Lebensjahr?

Bei jüngeren mehr als bei älteren?

Bei verschieden intelligenten Personen gleich?

In fluider wie in kristalliner? 


\section{Probleme der Forschung}

IQ-Tests sind in Mitteln und Standardabweichung altersnormiert! Dadurch werden Anstiege und Varianzänderungen verdeckt.

Was ist ein Maß fluider Intelligenz, was eines kristalliner?

Intelligenz- und Schulleistungstests.

Qualität der Normstichproben und Normtabellen. 


\section{Bisherige Ergebnisse}

Kognitiver Kompetenzzuwachs

in ITBS, Klassenstufen 1 bis 9 , nach Gagné (2005)

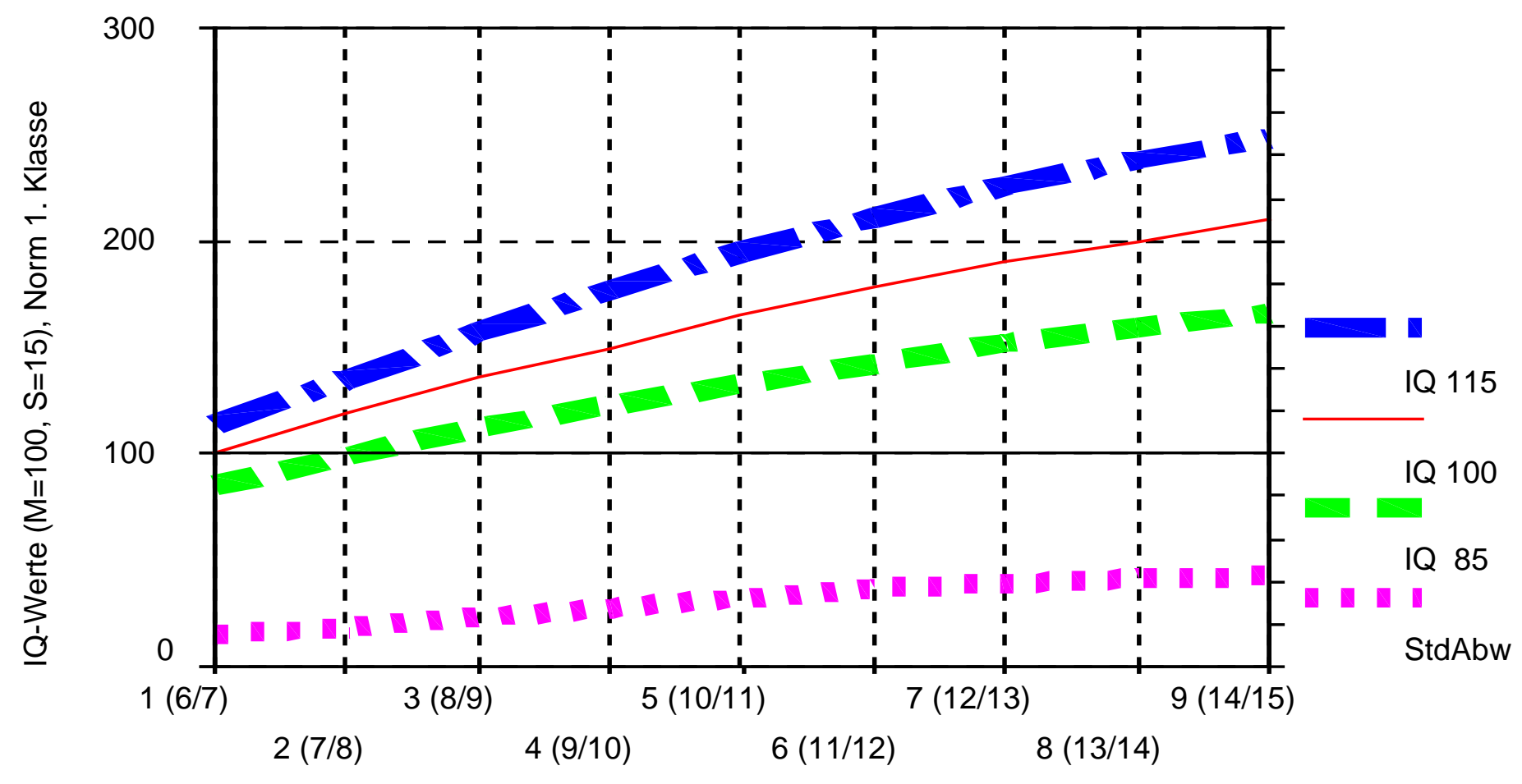

Klassenstufe (Alter)

Entwicklung kognitiver Kompetenzen im ITBS nach Gagné (2005) ITBS: lowa Tests of Basic Skills, schulwissensnah, Leseverständnis, Sprachkompetenz, Mathematik, Daten von 170.000 Schülern 


\section{Schulleistungsstudien (schulnahe kognitive Kompetenzen)}

TIMSS, IGLU, PISA, ASVAB.

Im Schnitt je Schuljahr 40-50 Punkte (äquivalent IQ=6,0-7,5), bei jüngeren mehr.

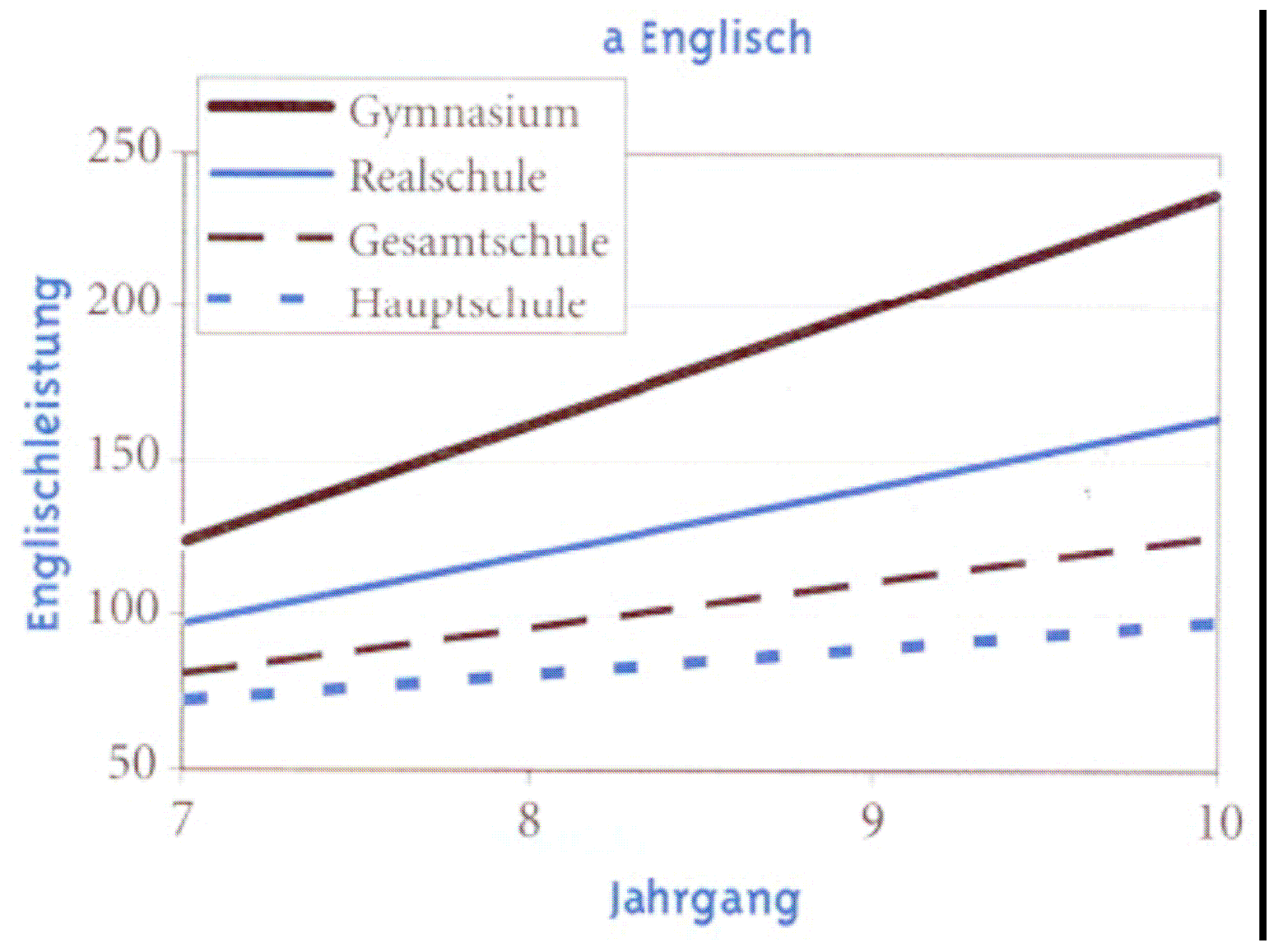

Nach Schulformen (BIJU-Studie; Köller \& Baumert, 2008, S. 742). 


\section{Testauswertungen}

\section{Methode}

Normtabellen von Intelligenztests mit identischen Aufgaben für verschiedene Altersstufen auswerten:

Wenn bspw. Kinder mit 12 Jahren in den Normen für 11-Jährige vier IQ-Punkte besser abschneiden, dann spricht dies für ein Intelligenzwachstum von vier IQ-Punkten.

- Für IQ 100, 85 und 115.

- Je IQ-Level zwei Werte.

- Zuwachs.

- Glättung. (Zum Teil seltsame Ergebnisse!)

- Kategorisierung in schulfern (fluide) und schulnah (kristallin).

- 11 verschiedene Tests. Nur deutsche Tests. Die erreichbar.

- Nicht Staffeltests (KFT, HAWIK, AID, K-ABC). 


\section{Ergebnisse}

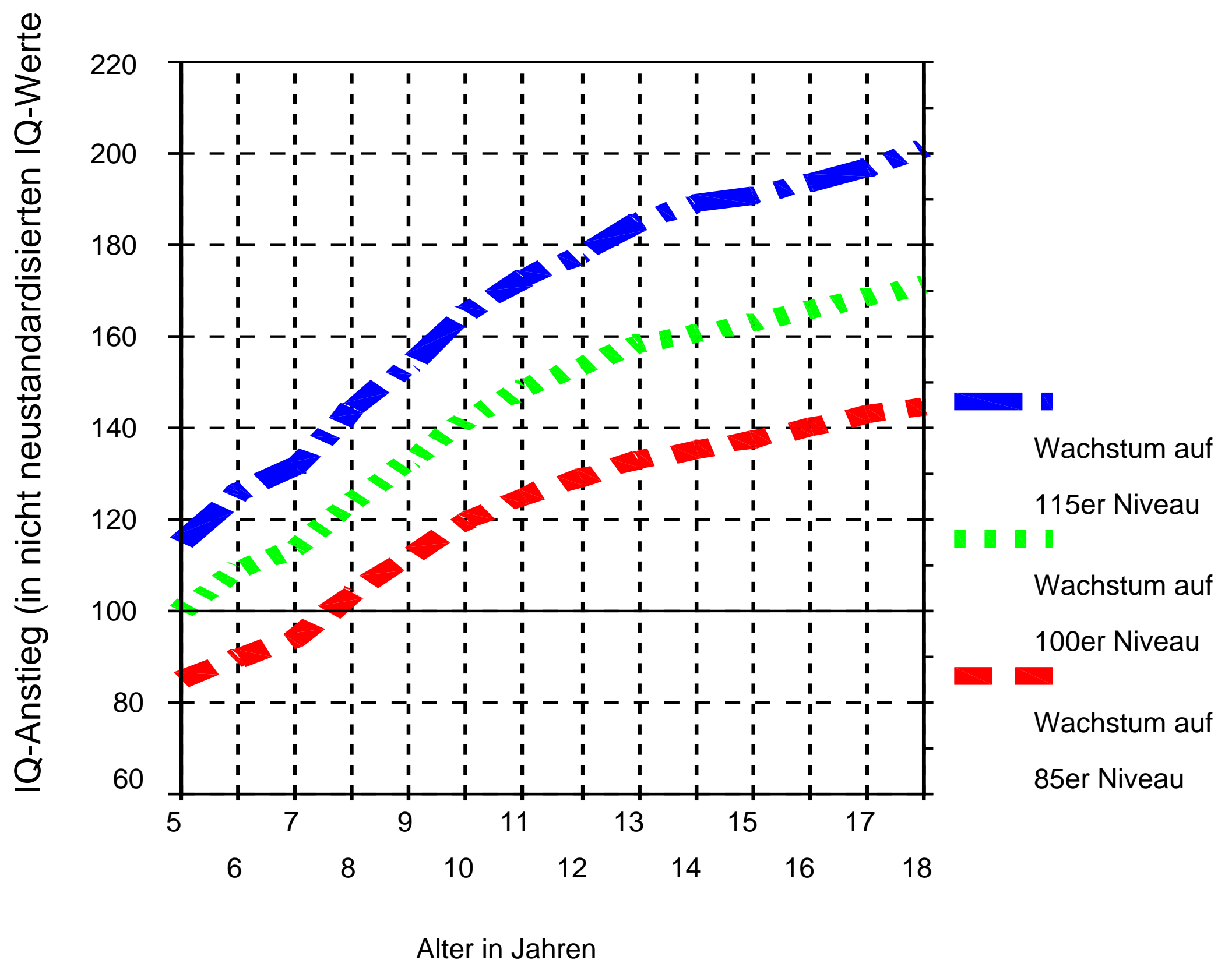


In Normauswertung im Schnitt um 5,58 IQ-Punkte je Lebensjahr.

Auf höherem Fähigkeitsniveau ist der Zuwachs größer

(Schereneffekt):

IQ-115-Niveau: 6,64 IQ-Punkte.

IQ-100-Niveau: 5,49 IQ-Punkte.

IQ-085-Niveau: 4,60 IQ-Punkte.

Der Zugewinn pro Jahr ist bei jüngeren Kindern höher:

Sechs- bis Neunjährige: $\quad 8,18$ IQ-Punkte.

Zehn- bis Vierzehnjährige: 5,77 IQ-Punkte.

Fünfzehn- bis Achtzehnjährige: 2,73 IQ-Punkte.

Der Zuwachs ist in der Schulzeit in der kristallinen Intelligenz höher:

CFT-kristalline Intelligenz: 5,05 IQ-Punkte. (Wortschatz, Zahlenfolge.)

CFT-fluide Intelligenz: $\quad 3,51$ IQ-Punkte. 


\section{Querschnittliche Studien}

\section{Methode 1}

- Gymnasiasten erhalten KFT, altersgemäße und die Aufgaben der davor liegenden Klassenstufe. Leichte Zeitverlängerung.

- Vergleich der IQ-Werte der „richtigen“ Klasse und davor.

- 5. bis 12 . Klassen, Steiermark, $N=149$.

- Kategorisierung in schulfern (N1, N2) und schulnah (V1, V4, Q2, Q3).

- Glättung. (Ergebnisse sind nicht nur stichproben-, sondern auch normtabellenabhängig.) 


\section{Ergebnisse 1}

Kognitiver Kompetenzzuwachs

KFT fluide vs. kristallin

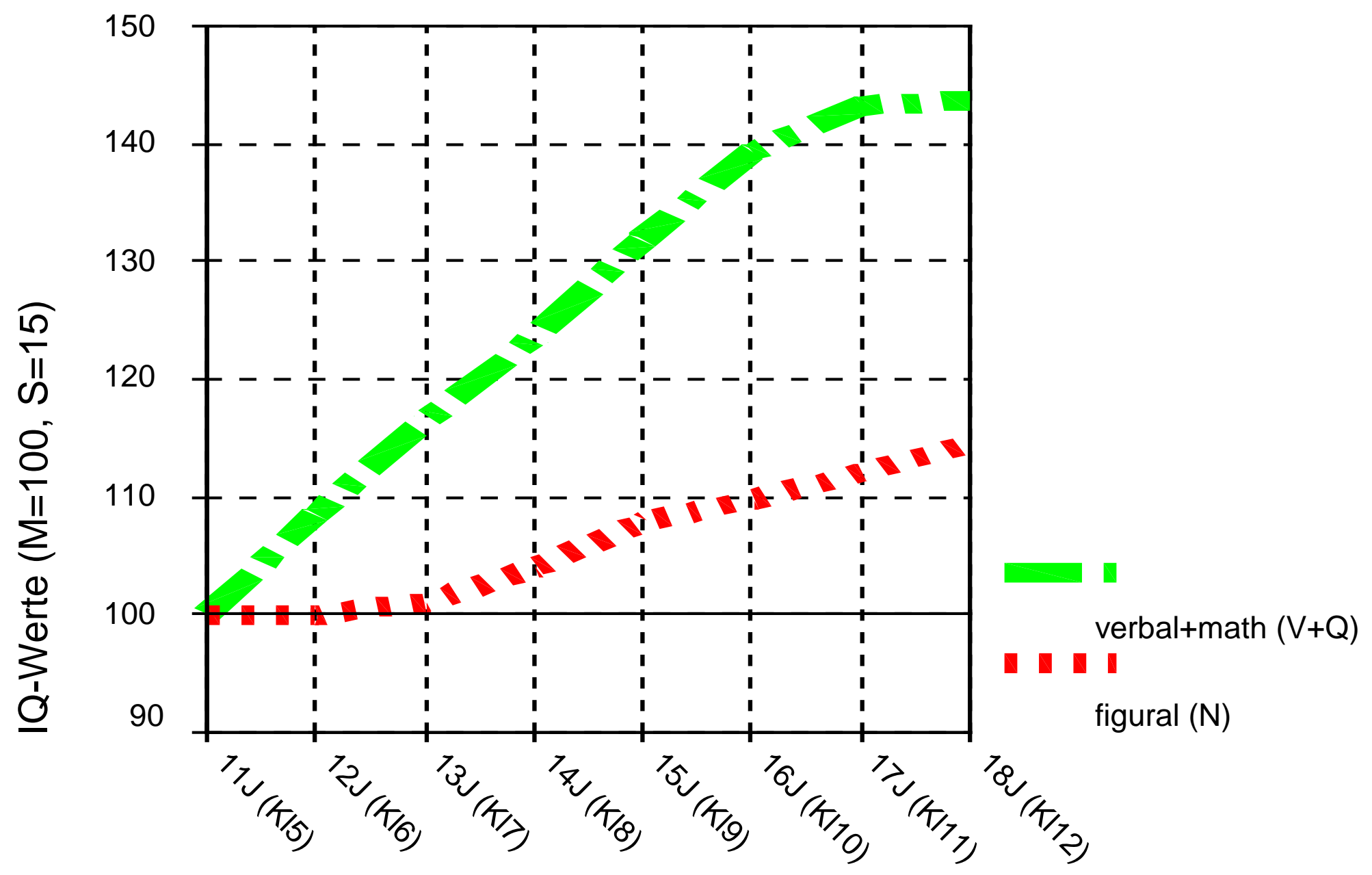


Zuwachs im Schnitt um 4,85 IQ-Punkte je Schuljahr.

Der Zugewinn pro Jahr ist bei jüngeren Kindern höher:

11-14 Jahre (Klasse 5-8): 5,69 IQ-Punkte.

14-18 Jahre (Klasse 8-12): 4,22 IQ-Punkte.

Der Zuwachs ist in der Schulzeit in der kristallinen Intelligenz höher:

KFT-kristalline Intelligenz: 6,24 IQ-Punkte.

KFT-fluide Intelligenz: $\quad$ 2,08 IQ-Punkte. 


\section{Methode 2}

- Gymnasiasten erhalten KFT, altersgemäße und die Aufgaben der darüber liegenden Klassenstufe. Keine Zeitverlängerung.

- Vergleich der IQ-Werte der „richtigen“ Klasse und darüber.

- 5. bis 13. Klassen, Baden-Württemberg, $N=521$.

- Positiv selegierte Stichprobe (Baden-Württemberg, Hälfte Begabtenfördergymnasium, 66\% der Väter und 46\% der Mütter hatten akademischen Abschluß, mittlerer IQ um 124 [127 vs. 116]).

- Kategorisierung in schulfern (N1, N2) und schulnah (V1, V4, Q2, Q3). 


\section{Ergebnisse 2}

Zuwachs im Schnitt um 6,10 IQ-Punkte je Schuljahr.

Der Zugewinn pro Jahr ist bei jüngeren Kindern höher:

11-14 Jahre (Klasse 5-8): 8,14 IQ-Punkte.

14-18 Jahre (Klasse 9-12): 4,05 IQ-Punkte.

Der Zuwachs ist in der Schulzeit in der kristallinen Intelligenz höher:

KFT-kristalline Intelligenz: 6,83 IQ-Punkte.

KFT-fluide Intelligenz: $\quad$ 4,62 IQ-Punkte.

Auf höherem Fähigkeitsniveau ist der Zuwachs größer

(Schereneffekt):

IQ-115-Niveau: 6,19 IQ-Punkte.

IQ-100-Niveau: 6,07 IQ-Punkte.

IQ-094-Niveau: 5,36 IQ-Punkte. 


\section{Längsschnittliche Studie}

\section{Methode}

- Gymnasiasten erhalten KFT, altersgemäße und die Aufgaben der darüber liegenden Klassenstufe. Keine Zeitverlängerung.

- Welchen IQ-Wert hatten die Schüler ein Jahr zuvor in den Aufgaben für die Klassenstufe damals darüber? Vergleich mit dem jetzigen.

- Wechsel von KFT-Parallel-A- und KFT-Parallel-B-Formen.

- 5. bis 13. Klassen, Baden-Württemberg, $N=461$.

- Positiv selegierte Stichprobe (Baden-Württemberg, Hälfte Begabtenfördergymnasium, 66\% der Väter und 46\% der Mütter hatten akademischen Abschluß, mittlerer IQ um 124 [127 vs. 116]).

- Kategorisierung in schulfern (N1, N2) und schulnah (V1, V4, Q2, Q3). 


\section{Ergebnisse}

\section{Kognitiver Kompetenzzuwachs}

KFT fluide vs. kristallin

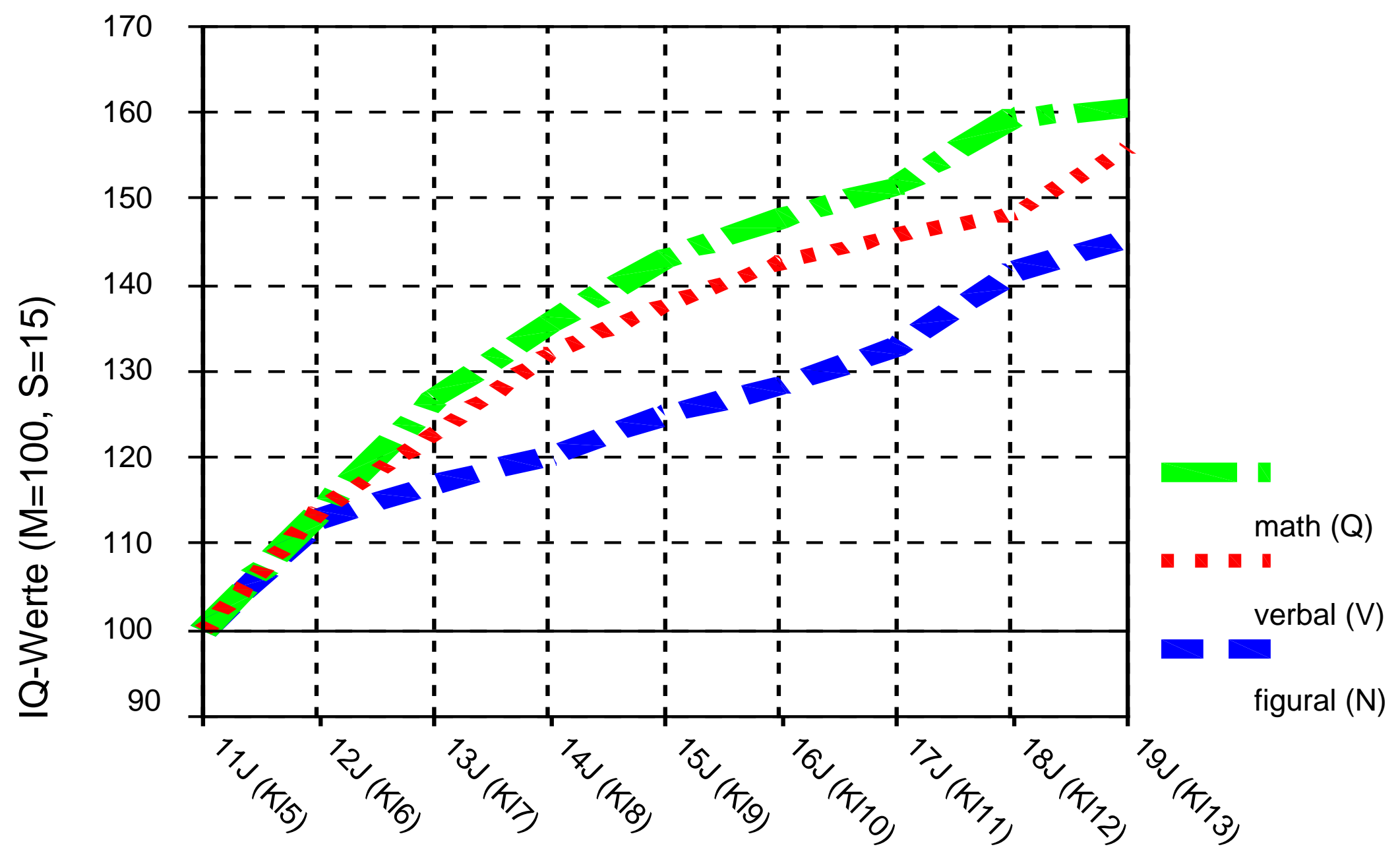


Zuwachs im Schnitt um 6,74 IQ-Punkte je Schuljahr.

Der Zugewinn pro Jahr ist bei jüngeren Kindern höher:

11-14 Jahre (Klasse 5-8): 9,78 IQ-Punkte.

14-18 Jahre (Klasse 9-12): 4,91 IQ-Punkte.

Der Zuwachs ist in der Schulzeit in der kristallinen Intelligenz höher:

KFT-kristalline Intelligenz: 7,28 IQ-Punkte.

KFT-fluide Intelligenz: $\quad$ 5,66 IQ-Punkte.

Auf höherem Fähigkeitsniveau ist der Zuwachs größer

(Schereneffekt):

IQ-115-Niveau: 6,90 IQ-Punkte.

IQ-100-Niveau: 6,84 IQ-Punkte.

IQ-094-Niveau: 5,53 IQ-Punkte. 


\section{Diskussion und Ausblick}

Die Intelligenz steigt im Mittel der vier Studien durchschnittlich um 5,62 IQ-Punkte je Lebensjahr an.

$\rightarrow$ Robustes Ergebnismuster, wenig spezifische Methodeneffekte.

Auf höherem Fähigkeitsniveau ist der Zuwachs größer (Schereneffekt):

IQ-115-Niveau: 6,59 IQ-Punkte.

IQ-100-Niveau: 5,97 IQ-Punkte. IQ-085-Niveau: 5,02 IQ-Punkte.

$\rightarrow$ Kombination von umweltlichen und genetischen Effekten. 
Der Zuwachs ist in der Schulzeit in der kristallinen Intelligenz höher: Kristalline Intelligenz: 6,12 IQ-Punkte. Fluide Intelligenz: $\quad 3,58$ IQ-Punkte.

$\rightarrow$ Schul-, Wissens- und Umwelteffekte (und der Fähigkeit, sie zu nutzen).

Der Zugewinn pro Jahr ist bei jüngeren Kindern höher:

Sechs- bis Neunjährige: $\quad$ 8,18 IQ-Punkte.

Zehn- bis Vierzehnjährige: $\quad$ 5,77 IQ-Punkte.

Fünfzehn- bis Achtzehnjährige: 2,73 IQ-Punkte.

$\rightarrow$ Negativ beschleunigt. Wegen Abhängigkeit von Höhe des Ausgangswertes? Neurobiologische Limits? Achtung: Skalenprobleme (Bezugsjahr der Normierung). 
Bei Eltern mit unterschiedlichem Bildungsgrad und unterschiedlicher Intelligenz verschieden?

In Ländern mit unterschiedlicher Bildungshöhe, Schulqualität und mittlerer kognitiver Fähigkeit verschieden?

Einheimische vs. Migranten (verschiedener Herkunft).

Nach Schulform.

Vor 30-50 Jahren so wie heute?

Neuere Verfahren und mehr nehmen!

Latent Growth Modelling? 


\section{Literatur}

Rindermann, H. (2011). Intelligenzwachstum in Kindheit und Jugend. Psychologie in Erziehung und Unterricht, 58(3), 210-224. [darin alle weiteren Referenzen] 